\title{
Klinische Forschung in der Schweiz - die Rolle der Clinical Trial Units
}

Tay, F ; Senti, G ; Althaus, B

\begin{abstract}
Am Beispiel der Universitätsspitäler Zürich und Basel wird in den folgenden zwei Beiträgen gezeigt, welche Aufgaben Clinical Trial Units haben und welche Dienstleistungen für Forscher, Ärztinnen und Ärzte sie bieten. Auch Angebote zur Aus- und Weiterbildung sind wichtiger Bestandteil ihrer Arbeit.
\end{abstract}

Posted at the Zurich Open Repository and Archive, University of Zurich

ZORA URL: https://doi.org/10.5167/uzh-60904

Journal Article

Published Version

Originally published at:

Tay, F; Senti, G; Althaus, B (2011). Klinische Forschung in der Schweiz - die Rolle der Clinical Trial Units. Schweizerische Ärztezeitung, 92(50):1966-1969. 


\title{
Klinische Forschung in der Schweiz - die Rolle der Clinical Trial Units
}

\author{
Am Beispiel der Universitätsspitäler Zürich und Basel wird in den folgenden zwei \\ Beiträgen gezeigt, welche Aufgaben Clinical Trial Units haben und welche Dienst- \\ leistungen für Forscher, Ärztinnen und Ärzte sie bieten. Auch Angebote zur Aus- und \\ Weiterbildung sind wichtiger Bestandteil ihrer Arbeit.
}

Fabian Tay a, Gabriela Senti ${ }^{b}$ Beat Althausc

a Dr. med., Prüfarzt, Clinical Trials Center Zürich

b PD Dr. med., Leiterin Clinical Trials Center Zürich

c Dr. med., Weiterbildungsstättenleiter FMH, Clinical Trials Center Zürich
Korrespondenz: Dr. med. Beat Althaus Clinical Trials Center Zentrum für Klinische Forschung Universität und UniversitätsSpital Zürich Rämistrasse 100 CH-8091 Zürich
Clinical Trial Units (CTUs) sind universitäre Organisationseinheiten, die zur Durchführung von klinischen Studien mit speziell dafür qualifizierten Mitarbeitern ins Leben gerufen wurden. In der Schweiz besteht eine Zusammenarbeit zwischen den Universitäten, den Universitätsspitälern und dem Schweizerischen Nationalfonds mit dem Ziel, die akademische Forschung zu fördern und zu verbessern. Die Dachorganisation ist die Swiss Clinical Trial Organisation (SCTO) [1]. In der Schweiz gibt es sechs dieser akademischen klinischen Forschungszentren. Diese vom Schweizerischen Nationalfonds unterstützten Clinical Trial Units (CTUs) befinden sich an den Universitätsspitälern Basel, Bern, Genf, Lausanne, Zürich sowie am Kantonsspital St. Gallen [2-7].

Am Beispiel des Clinical Trials Center (CTC) der Universität und des UniversitätsSpitals Zürich werden im Folgenden die einzelnen Aufgaben einer solchen CTU erläutert.

\section{Die Aufgaben einer CTU am Beispiel des CTC} Das CTC unterstützt alle Forschungsgruppen des UniversitätsSpitals Zürich bei der Planung und Durchführung klinischer Studien gemäss Schweizerischem Heilmittelgesetz und internationalen Good Clinical Practice Standards (GCP-Standards) [8]. Zudem hat das CTC vom Zürcher Regierungsrat das Mandat erhalten, als Kompetenzzentrum die Qualität der klinischen Forschung und somit die hochspezialisierte Medizin flächendeckend im ganzen Kanton Zürich zu fördern und die der medizinischen Fakultät assoziierten Spitäler in ihre Dienstleistungen mit einzubeziehen.

Deshalb hat sich das CTC zum Ziel gesetzt, die Infrastruktur, die Kompetenz und das Qualitätsniveau auf dem Gebiet der klinischen Forschung fortlaufend anzuheben. Damit soll die Wettbewerbsfähigkeit im nationalen und internationalen Vergleich längerfristig gewährleistet werden.

Ferner sieht das CTC seine Aufgabe darin, Assistenzärztinnen und -ärzte zum Facharzttitel für Pharmazeutische Medizin weiterzubilden, sowie Naturwissenschaftler, Studienkoordinatorinnen, Monitorinnen

\section{La recherche clinique en Suisse - le rôle des Clinical Trial Units}

Unités universitaires dédiées à la recherche clinique, les Clinical Trial Units (CTU) disposent d'un personnel spécialement qualifié et sont regroupées dans un réseau sous la houlette de l'organisation faîtière Swiss Clinical Trial Organisation (SCTO). Sur la base d'un exemple, celui du Clinical Trial Center (CTC) de Zurich, les auteurs décrivent les tâches des CTU et présentent les services auxquels peuvent faire appel les chercheurs en cas de besoin. Proposant différentes possibilités de formation comme des cours sur les Good Clinical Practice (GCP) ou un cursus universitaire sous forme d'un Certificate of Advanced Studies (CAS) in Clinical Trial Management, le CTC est également un établissement de formation postgraduée en médecine pharmaceutique de catégorie $\mathrm{A}$ reconnu par la FMH. En définitive, les CTU sont des centres de compétences qui contribuent à la qualité de la recherche clinique et à la consolidation de la place Suisse en matière de recherche.

und alle weiteren in der klinischen Forschung tätigen Mitarbeiter aus- und weiterzubilden.

Das CTC verfügt als eigene Organisationseinheit im UniversitätsSpital Zürich über eine ambulante und eine stationäre klinische Forschungsstation (mit Phase-I-Unit). Diese Infrastruktur erlaubt es CTC-Studienärzten, selbständig probanden- und patientenorientierte Forschungsprojekte durchzuführen.

\section{Leistungsangebot des CTC}

Das CTC bietet für klinische Forschungsprojekte die Machbarkeitsabklärung, Planung, Koordina- 
tion, Durchführung, sowie das Datamanagement und Monitoring/Auditing nach geltenden Gesetzen und international anerkannten Qualitätsstandards an.

Dies umfasst alle Dienstleistungen im Bereich der klinischen Forschung für sämtliche durch akademische Forscher initiierten Studien (sog. Investigator Initiated Trials, IITs). Ein breitausgebildetes Spezialistenteam unterstützt dabei die universitären Forschungsgruppen entsprechend deren Bedürfnissen.
Während davon in erster Linie das Universitätsspital Zürich und assoziierte Spitäler profitieren, können im Rahmen des kantonalen Mandats weitere Spitäler mit öffentlichem Auftrag daraus Nutzen ziehen. Zudem bestehen Kollaborationen mit Hochschulen sowie mit der pharmazeutischen und Medizintechnik-Industrie.

Durch das breite Angebot bietet das CTC Hilfestellung zu einer effizienten Durchführung sowie einer kompetenten Qualitätskontrolle von klinischen

\section{Das CTC bietet für Forschungsprojekte die Machbarkeitsabklärung, Planung, Koordination, Durchführung sowie das Datamanagement und Monitoring/Auditing.}

Die ärztlichen und wissenschaftlichen Mitarbeiter des CTC unterstützen klinische Forschungsteams bei der Studienvorbereitung und Durchführung der klinischen Studien. Dazu gehören z. B. die Beratung beim Erstellen von Prüfprotokollen, von elektronischen Prüfbögen und allen erforderlichen Studiendokumenten. Sie geben Hilfestellung bei der Vorbereitung für die Eingabe eines Projekts bei der Ethikkommission oder der Notifikation bei der Behörde Swissmedic. Ausserdem führt das CTC Qualitätskontrollen von laufenden Studien in Form von Monitoring oder Audits durch, übernimmt die Datenevaluation oder bietet Unterstützung bei der regelmässigen Berichterstellung an Behörden. Sehr gefragt sind fachspezifische Beratungen in allen regulatorischen und finanziellen Fragen, bei denen Forscher häufig gerne Hilfe in Anspruch nehmen.

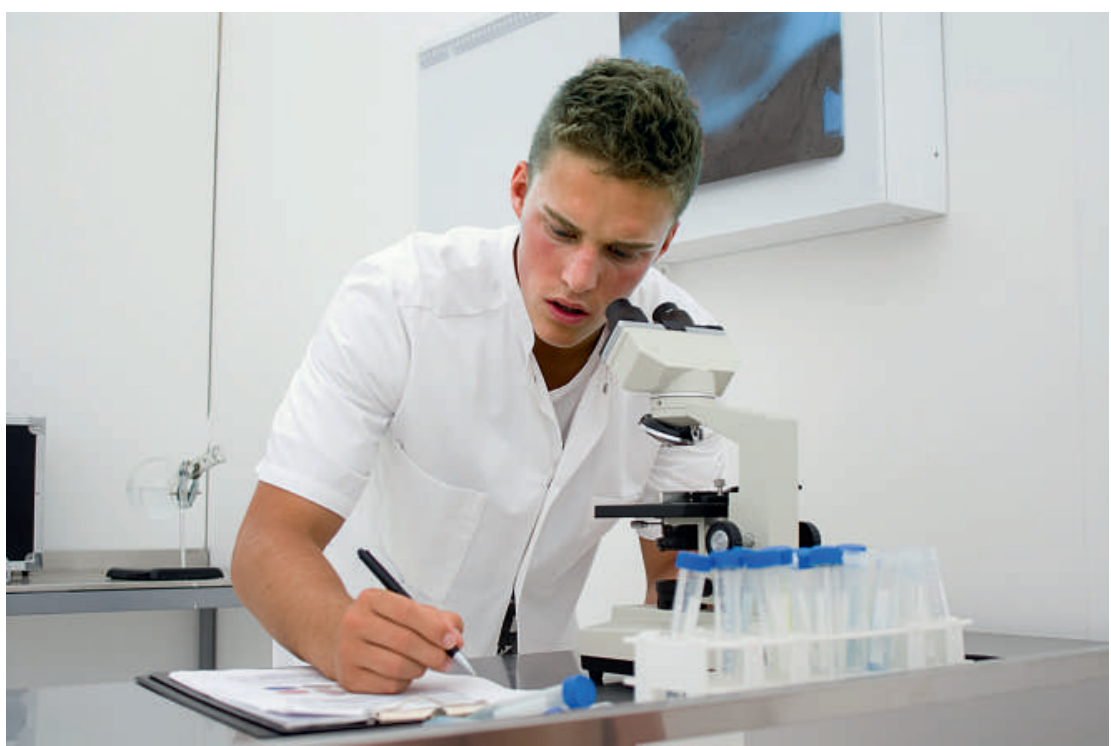

Clinical Trial Units unterstützen Forscher bei der Planung und Durchführung klinischer Studien.
Forschungsprojekten. Dabei hat das CTC in diesem Jahr selbst wichtige Prozesse zur Qualitätsverbesserung durchlaufen: Das CTC ist als erste CTU ein nach ISO 9001 zertifiziertes Kompetenzzentrum für klinische Forschung. Zudem wurde es wie alle eingangs erwähnten CTUs einer Swissmedic-Inspektion unterzogen, die sehr erfolgreich abgelaufen ist.

\section{Weiterbildungsaufgaben}

Die Weiterbildung hat am CTC einen sehr grossen Stellenwert. Das CTC bietet verschiedene Kurse im Bereich der klinischen Forschung an, insbesondere Good-Clinical-Practice-Kurse (GCP), die Swissmedic akkreditiert sind. Die GCP-Kurse sind entsprechend den Anforderungen von Swissmedic [9] in verschiedene Module unterteilt. Die Teilnahme am Grundkurs ist für alle am UniversitätsSpital Zürich neu eintretenden Ärztinnen und Ärzte und an klinischen Studien beteiligte Personen obligatorisch. Allein im Jahr 2010 wurden 32 GCP-Kurse durchgeführt mit insgesamt 670 Teilnehmern.

Darüber hinaus wird am CTC ein universitärer Zertifikatsstudiengang angeboten. Ein erfolgreicher Abschluss führt $\mathrm{zu}$ einem Certificate of Advanced Studies UZH in Clinical Trial Management. Dieser berufsbegleitende modulare Lehrgang richtet sich an ärztliches wie auch an nicht ärztliches Studienpersonal, das an klinischen Studien bereits mitwirkt oder sich in diesen Bereich einarbeiten möchte. Er vermittelt den Teilnehmern fundierte theoretische und praktische Kenntnisse zu Planung und Organisation klinischer Studien. Wesentliche Inhalte sind hierbei die Gebiete Ethik und Recht, Biostatistik, Grundlagen des Datenmanagements, Klinisches Monitoring und Studienassistenz, Ergebnisbewertung sowie Berichterstellung und Publikation. Der Studiengang gilt zudem als Nachweis für eine Weiterbildung gemäss den Anforderungen von Swissmedic für Principal Investigator-Sponsor im Sinne der Verordnung über klinische Versuche mit Heilmitteln (VKlin) 
[10] und des Schweizerischen Heilmittelgesetzes (HMG) [11].

Hervorzuheben ist das CTC als Weiterbildungszentrum für Pharmazeutische Medizin. Das CTC wurde im Februar 2011 als bis dahin einzige universitäre Weiterbildungsstätte von der FMH als Kategorie A akkreditiert. Es bietet seinen Assistenzärzten eine strukturierte Weiterbildung im Rahmen eines definierten Weiterbildungskonzepts auf dem Weg zum eidgenössischen Facharzttitel für Pharmazeutische Medizin. der klinischen Forschung bieten die CTUs neben Räumlichkeiten und Infrastruktur eine kompetente Beratung, breitgefächerte Dienstleistungen von der Konzeption bis zum Abschlussbericht einer klinischen Studie an. Die CTUs arbeiten mit Klinikern und Grundlagenforschern zusammen und setzen sich für innovative klinische Forschungsprojekte ein. Die ärztlichen und wissenschaftlichen Mitarbeiter der CTUs beraten und unterstützen Kliniker und Forscher in allen spezifischen Fragen zu klinischen Studien bezüglich der praktischen Um-

\section{«Sehr gefragt sind fachspezifische Beratungen in allen regulatorischen und finanziellen Fragen, bei denen Forscher häufig gerne Hilfe in}

\section{Anspruch nehmen.»}

Ziel der praktischen Weiterbildung am CTC ist, einen breiten Überblick über die unterschiedlichen Aspekte der Pharmazeutischen Medizin zu vermitteln und ein fundiertes Verständnis für ineinandergreifende Arbeits- und Verantwortlichkeitsgebiete zu erzeugen. Darüber hinaus soll in der Weiterbildungsstätte ein ethisch-motiviertes Grundverständnis für die Rolle des Arztes in der Pharmazeutischen Medizin vermittelt werden.

Anders als in industriellen Weiterbildungsstätten lernen die Assistenzärztinnen und -ärzte, selbständig in der ambulanten und stationären klinischen Forschungsstation probanden- und patientenorientierte Forschungsprojekte der Phasen I-IV zu planen, vorzubereiten und durchzuführen. Weiter werden Tätigkeiten in den Bereichen medizinisch-wissenschaftliche Information, Arzneimittelsicherheit und Sozioökonomie des Gesundheitssystems praktisch geschult. Ausserdem stehen Möglichkeiten zur Mitarbeit in der präklinischen Forschung offen. Derzeit stehen fünf Assistenzärzte in Weiterbildung zum Facharzt für Pharmazeutische Medizin und sieben Naturwissenschaftler in Weiterbildung zum SwAPP Diplom.

\section{Schlusswort}

Klinische Studien dienen der Entwicklung neuer oder der Verbesserung vorhandener Medikamente und Behandlungsmethoden, was eine systematische Untersuchung an Probanden und Patienten voraussetzt. An deren Planung, Durchführung und Auswertung werden strenge wissenschaftliche, ethische und rechtliche Anforderungen gestellt. Eine qualitativ hochwertige, akademische patientenorientierte Forschung ist die Kernaufgabe von Clinical Trial Units. Durch die langjährige Erfahrung der Mitarbeiter der CTUs in allen Teilaspekten setzung einer wissenschaftlichen Fragestellung, Idee oder Erfindung. Entsprechende Forschungsprojekte sollen sich in Zukunft vermehrt darin auszeichnen, neueste Erkenntnisse aus der klinischen Forschung möglichst rasch und sicher in den klinischen Alltag zu bringen. Damit kann die hohe Qualität des Forschungsstandorts Schweiz gesichert werden.

\section{Referenzen}

1 Swiss Clinical Trial Organisation: www.scto.ch

2 Study Coordination Center Basel: www.clinicaltrialunit.ch

3 CTU Bern: www.ctu-bern.ch

4 Centre de recherche clinique aux Hôpitaux Universitaires de Genève: crc.hug-ge.ch

5 Centre de recherche clinique Lausanne: chuv.ch/ chuv_home/recherche/chuv_recherche-infospratiques/chuv_recherche-infos-pratiques-crc.html

6 Clinical Trials Center CTC Zürich: www.ctc-zkf.usz.ch

7 CTU St. Gallen: www.ctu.kssg.ch

8 ICH Topic E 6 (R1) Guideline for Good Clinical Practice: www.emea.europa.eu/docs/en GB/ document_library/Scientific_guideline/2009/09/ WC500002874.pdf

9 Swissmedic Anforderungen an die Ausbildung von Co-Prüfern, Hauptprüfern und Sponsor-Prüfern im Rahmen von klinischen Versuchen mit Heilmitteln www.swissmedic.ch/bewilligungen/00089/01100/ index.html

10 Verordnung über klinische Versuche mit Heilmitteln: www.admin.ch/ch/d/sr/c812_214_2.html

11 Bundesgesetz über Arzneimittel und Medizinprodukte (Heilmittelgesetz, HMG): www.admin.ch/ch/d/ sr/c812_21.html 